\title{
Die chemische Zusammensetzung menschlichen Chylusfettes. Von
}

\author{
Demonstrator Franz Erben.
}

(Aus dem Laboratorium für medicinische Chemie in Wien.)

(Der Redaction zugegangen am 11. Juli 1900.)

Die seltene Gelegenheit, einen Fall von Chylurie durch längere Zeit beobachten zu können, bot mir die Möglichkeit, eine grössere Menge menschlichen Chylusfettes für eine chemische Analyse desselben sammeln zu können. Die Resultate der klinischen Beobachtung, die zu der Annahme drängte, dass der vorliegende Fall europäischer, nichtparasitärer Chylurie gleich der tropischen parasitären der Beimischung von Chylus zum Harne seine Entstehung verdankt, werden Dr. v. Stejskal und Dr. Franz in einer grösseren klinischen Arbeit niederlegen. Die vorliegenden Zeilen, die mit der klinischen Behandlung des Falles in keiner nähern Beziehung stehen, sind der chemischen Untersuchung des Harnfettes gewidmet.

Es sei mir aber gestattet, an erster Stelle unter Beiziehung der klinischen Beobachtungen die Berechtigung, das Harnfett als Chylusfett anzusprechen, zu beweisen, wobei ich aber der Vollständigkeit der Beweisführung halber die zu derselben nothwendigen Daten der chemischen Analyse des Harnfettes antecipiren muss. Die Ergebnisse der klinischen Versuche sind folgende:

1. Der Harn wurde nach zwölfstündigem Hungern bis auf minimale Spuren fettfrei. Zwei bis drei Stunden nach der ersten Nahrungsaufnahme konnte Fett wieder deutlich nachgewiesen werden. 
2. Das gleiche Verhalten wurde beobachtet, wenn Patient eine fast vollkommen fettfreie Nahrung, die aber für die Ernährung genügende Mengen Eiweiss und Kohlehydrate dem Organismus zuführte, erhielt. Inanition war also bei diesem Versuch vollständig ausgeschlossen.

3. Mit Sudan III. gefärbtes Fett, per os eingeführt, erschien nach kürzester Zeit im Harn wieder. Im intensiv roth gefärbten Aetherextract dieses Harnes war Sudan nachweisbar.

4. Der Fettgehalt des Blutes beträgt $0.378 \%$, ist also sicher nicht vermehrt. Der Fettgehalt des Harnes hingegen schwankt zwischen weiten Grenzen, z. B. fand ich in der Eiweissperiode $0,564 \%$ bis $2,158 \%$.

Nun können zur Erklärung des Uebertrittes von Fett in den Harn nur folgende Möglichkeiten herangezogen werden:

a) Hochgradige fettige Degeneration der Epithelien des uropoetischen Systems mit Uebergang des Degenerationsfettes in den Harn (eventuell auch fettige Degeneration von Eiter),

b) Secretion des Fettes durch die Nierenepithelien, eventuell auch durch heterologe Elemente, den Harn,

c) direkter Durchtritt von Fett aus dem Blute in

d) direkter Durchtritt fetthaltiger Lymphe (Chylus) in den Harn.

Fettige Degeneration, von vornherein schon unwahrscheinlich, ist auszuschliessen auf Grund der Versuche 2 und 3. Dieser letztere Versuch (Sudanfett) spricht gegen fettige Degeneration auf Grund einer, wie es scheint, von mehreren Seiten gemachten Beobachtung, von deren Richtigkeit ich mich selbst überzeugte, und die dahin geht, dass das im Fettgewebe abgelagerte Fett von Thieren, die mit Sudanfett längere Zeit gefüttert ·worden waren, sich roth färbte, während pathologisch durch fettige Degeneration (z. B. bei Phosphorvergiftung) auftretendes Fett ungefärbt bleibt. Fettige Degeneration ist aber auch ausgeschlossen wegen der grossen Menge des täglich ausgeschiedenen Fettes $(5-30 \mathrm{~g})$ bei der langen Dauer des Zustandes, ferner wegen des Umstandes, 
dass bei gewöhnlicher Kost das Fett fest, bei Lipaninfütterung aber flüssig war, und endlich aus den Ergebnissen der chemischen Untersuchung des Harnfettes. Denn nach den Beobachtungen von Lindemann ${ }^{1}$ ) hat Degenerationsfett eine hohe Säurezahl $(18,35 d . h$. ist reich an freien Fettsäuren), während die Säurezahl unseres Fettes sehr klein ist $(3,5)$.

Gegen die Annahme einer Secretion des Fettes spricht erstens das prompte Ausbleiben beim Hungern, aber auch dann, wenn nur fettfreie Nahrung gereicht wurde (Versuche 1 und 2), zweitens aber auch die Thatsache, dass bei Einfuhr von Lipanin das bei gewöhnlicher Spitalskost feste Fett sofort einem flüssigen Platz machte (siehe weiter unten). Es liegt in dieser prompten Aenderung der Beschaffenheit des Harnfettes durch die des Nahrungsfettes zweifellos ein Moment gegen die Annahme einer Secretion. Es widerspräche allen physiologischen Erfahrungen über die Secretion, wenn in kürzester Zeit nach Aufhören bloss der Fettzufuhr die Secretion vollständig versiegen, resp. bei qualitativer Aenderung des Nahrungsfettes das secernirte Fett plötzlich seine physikalischen Eigenschaften ändern sollte. Mit der Annahme einer Secretion wäre eben nur ein allmähliches, langsames, erst nach dem Verbrauche allen im Körper aufgespeicherten Reservemateriales vollständiges Verschwinden, resp. im zweiten Falle, wenn überhaupt, so ein allmählicher Uebergang vereinbar. Es ist daher die Annahme einer Secretion des Fettes vollständig von der Hand zu weisen.

$\mathrm{Ob}$ die Annahme Eggels, ${ }^{2}$ ) dass das Harnfett aus dem Blute stamme, aus dem es durch Poren, die neben Blutserum wohl feinsten Fetttröpfchen, aber nicht rothen Blutkörperchen die Passage gestatten, in die harnleitenden Wege einfach durchfiltrire, richtig sei, muss eine Bestimmung des Fettgehaltes des Blutes lehren. Das Blut des Eggel'schen Falles wurde von Hoppe-S eyler ${ }^{3}$ ) analysirt. Der Aetherextract (Fett und Lecithin) beträgt $0,676 \%$, der Lecithingehalt $0,348 \%$.

1) Ueber das Fett des normalen und fettig entarteten Herzmuskels Zeitschrift f. Biol. Bd. 38.

2) Archiv für Klinische Medicin, Bd. 4.

3) Medicinisch chemische Untersuchangen, Heft 4, 1869. 
Der Blut-Fettgehalt, der allein in Betracht kommt $(0,328 \%$, ist also erheblich niedriger als der Harnfettgehalt $(0,687)$. Das Gleiche gilt für unsern Fall; der Fettgehalt des Blutes beträgt $0,378 \%$, der des Harnes z. B. in der Eiweissperiode im Mittel 1,032\% \%. Aus dem Vergleiche dieser Zahlen ergibt sich, dass ein einfacher Durchtritt fetthaltigen Serums in diesen Fällen unmöglich angenommen werden kann, denn dann müsste der Fettgehalt des Harnes gleich oder höchstens unwesentlich höher (wegen der Resorption von Harnwasser in der Blase) sein als der des Blutes. Die obigen Zahlen könnten nur durch eine Secretion d. h. selective Ausscheidung von Fett aus dem Blute erklärt werden, was wir oben mit zwingenden Gründen ausgeschlossen haben.

Es bleibt also nur die letzte Möglichkeit, die Herkunft des Fettes aus der Lymphe. Dass das Fett aber aus der gewöhnlichen Körperlymphe mit $0,2-0,4 \%$ Fett nicht stammen kann, erhellt aus denselben Gründen, die wir gegen die Annahme c (aus dem Blute) angeführt haben und die schon Brieger gewürdigt hat. Es kann nur Beimischung von fetthaltiger Lymphe, also der des Darmes (Chylus), angenommen werden, wobei allerdings aus anatomischen Gründen, Rückstauung des Chylus gegen die Nieren mit offener Communication der Chylus- mit den Harnwegen, vorauszusetzen ist. Für diese Annahme sprechen aber direkt die obenangeführten Versuche und die chemische Untersuchung des Harnes.

Nachdem so die Berechtigung, das Harnfett als Chylusfett anzusehen, bewiesen ist, wollen wir nun über die Resultate der chemischen Analyse desselben berichten. Menschliches Chylusfett ist bisher noch nicht genauer untersucht worden.

Spärliche Angaben finden sich bei Hensen, ${ }^{1}$ ) der die chylöse Flüssigkeit, die aus einer Lymphfistel am Präputium eines zehnjährigen Knaben sich entleerte, untersuchte. Dieselbe enthielt bis 2,25\% Fett. Bei fettarmer Diät sank der Fettgehalt auf $0,62 \%$. Das Fett enthielt 0,018-0,102\%

1) Pflüger's Archiv für Physiologie, Bd. 10, 1875. 
Cholesterin. Ausserdem konnte der Autor Seifen nachweisen, wie er vermuthet, Natronsalze der Oelsäure, Stearin- und Palmitinsäure.

Munk und Rosenstein ${ }^{1}$ ) untersuchten den Chylus aus einer Fistel am Oberschenkel. Das Fett, das sie bei Lipaninfütterung erhielten, war gelb, flüssig, mit einem Erstarrungspunkt unter $4^{\circ} \mathrm{C}$., es enthielt $2,4^{\circ} \%$ freie Fettsäure (als Oelsäure gerechnet), 1/14 - 1/15 Cholesterin, 1/14 Lecithin. Die Menge der Seifen machte 4,1-5\% der Fettmenge aus. Nach Hammeltalgfütterung war das Fett fest, gelb, der Schmelzpunkt desselben niedriger als der des Hammeltalges. Die Menge der Seifen betrug ${ }^{1 / 20}$ der des Fettes. Das Fett nach Einnahme von Erucasäure war fest, der Schmelzpunkt $25^{\circ}$, der Erstarrungspunkt 210; es enthielt 2,5\% freie Fettsäuren. 0,078\% Fettsäuren wurden aus den Seifen der chylösen Flüssigkeit gewonnen. Ueber das Fett, das bei gewöhnlicher Nahrung erhalten wurde, sind keine Angaben gemacht.

Die folgenden Autoren bearbeiteten Fälle von Chylurie:

$\left.\mathrm{Eggel}^{2}\right)$ stellte aus dem Aeterextract eines chylurischen Harnes Fettsäuren, resp. neutrale Fette, Cholestearin und Lecithin dar.

Brieger ${ }^{3}$ ) konnte bei einem Fall von Chylurie, der vollständig dem unserigen analog ist, aus $5^{1 / 2} \mathrm{l}$ Harn $8,93 \mathrm{~g}$ Fett extrahiren, das $0,189 \mathrm{~g}$ Cholesterin (ca. $2 \%$ ), Lecithin und $6,73 \mathrm{~g}$ Fettsäuren vom Schmelzpunkt $31^{\circ}$ enthielt.

Grimm ${ }^{4}$ ) beschrieb einen Fall von Filaria-Chylurie, bei dem eine Chylusblasenfistel constatirt wurde. Das Fett vom Schmelzpunkte $25^{\circ}$ bestand aus 55-67\% Palmitin und $33-45 \%$ Olein, bei Rapsölfütterung aus 17-22\% Glyceriden gesättigter und $83-78 \%$ Glyceriden ungesättigter Fettsäuren (Erucasäure).

Das Fett unseres Falles, das mir von dem Vorstande der

1) Virchow's Archiv, Bd. 123, 1890.

2) Archiv für Klinische Medicin, Bd. 4.

3) Diese Zeitschrift, Bd. IV, 1880.

4) Archiv für klinische Chirurgie, 32, 1886 und Virchow's Archiv, Bd. 111, 1888. 
medicinischen Abtheilung des k. k. Garnisonsspitales Nr. 1 in Wien, Herrn Regimentsarzt Dr. Franz, gütigst zur Analyse überlassen wurde, wurde aus mehreren getrennten Perioden in der Weise gewonnen, dass der möglichst frische Harn mit einer reichlichen Menge Aether ausgeschüttelt wurde. Die ätherische Lösung wurde durch Destillation vom Aether befreit. Nachdem so alles Fett einer Periode gesammelt war, wurde es im Wasserbade geschmolzen, bis es klar war, und dann durch ein trockenes Filter im Wärmekasten filtrirt.

Es blieb auf dem Filter ein auf demselben festhaftender, in der Wärme zäher, brauner Rückstand, der beim Erkalten fest und am Bruche undeutlich körnig wurde. Diese Substanz war in Aether löslich, wenn auch schwerer als das geschmolzene Fett. $\mathrm{Zu}$ einer vorläufigen Prüfung wurde sie mit wenig Aether von der grössten Menge anhaftenden Fettes befreit. Sie löste sich dann in Lauge zu einer opalisirenden Flüssigkeit vom Aussehen einer Seifenlösung vollständig auf. Mit Aether geschüttelt, gingen aus der Lösung die anhaftenden Fettspuren über, erst nach dem Ansäuern die fragliche Substanz. Durch dieses Verhalten war sie als Säure charakterisirt. Sie wurde daher in der Weise gereinigt, dass sie in verdünnter Sodalösung in der Wärme gelöst und, nachdem die Lösung durch mehrmaliges Ausschütteln mit Aether vollständig von Fett befreit war, aus derselben nach dem Ansäuren mit Schwefelsäure durch Ausschütteln mit Aether wieder gewonnen wurde.

So in reinem Zustande dargestellt, repräsentirte sie sich als eine lichtbraun gefärbte, ziemlich harte, undeutlich krystallinische, einem Fettsäurekuchen ähnliche Masse mit einem Schmelzpunkte von ca. $50^{\circ}$. Aus $100 \mathrm{~g}$ Fett wurden etwas über $1^{1 / 2} \mathrm{~g}$ in diesem reinen Zustande gewonnen. Zur chemischen Charakterisirung dieses Säuregemenges wurde eine Elementaranalyse und eine Molekulargewichtsbestimmung ${ }^{1}$ ) ausgeführt.

1) I. $0,0972 \mathrm{~g}$ Substanz gab $0,2582 \mathrm{~g} \mathrm{CO}_{\mathrm{z}}$ und $0,1022 \mathrm{~g} \mathrm{H}_{\mathbf{g}} \mathrm{O}$, II. 0,1060 > > $0,2817>\mathrm{CO}_{8}>0,1128>\mathrm{H}_{2} \mathrm{O}$. Die Substanz ist N-, P-, S-, und aschefrei.

III. 0,0982 g Substanz verbrauchte $3,0 \mathrm{ccm}$. KHO-Lösung $(1 \mathrm{ccm} .=$ 0,005808 g KHO). Die Verseifungszahl beträgt also 177,4. 
Die Substanz enthält:

I.

II.
C. $72,45 \%$
$72,49 \%$
$\mathrm{H} 11,69 \%$
$11,82 \%$
$015,86 \%$
$15,69 \%$.

Als mittleres Molekulargewicht wurde 316,2 gefunden.

Bei der Destillation mit Wasserdampf ging eine geringe Menge einer weissen, in Wasser unlöslichen, aus Aether krystallinisch sich abscheidenden, in Weingeist und Petroläther löslichen Substanz über, deren Schmelzpunkt zu $51,5^{\circ}$ bestimmt wurde.

Als kleinste Formel für diese Substanz ergibt sich aus den gefundenen Procentzahlen $\mathrm{C}_{6} \mathrm{H}_{12} \mathrm{O}$, welche Formel gemäss dem Molekulargewicht von $316 \mathrm{zu}$ verdreifachen ist. Der Verbindung $\mathrm{C}_{18} \mathrm{H}_{36} \mathrm{O}_{3}$ kommt ein Molekulargewicht von $300 \mathrm{zu}$. Es handelt sich also um ein Gemenge ${ }^{1}$ ) von Monoxystearinsäuren. Die aus demselben durch Destillation mit Wasserdampf erhaltene Substanz wird daher wahrscheinlich Stearolacton (Schmelzpunkt 51,2) sein.

Das, wie oben beschrieben, dargestellte Fett stammte aus vier verschiedenen Versuchsperioden.

Das Fett der Periode I war fest, dunkelgelb, sein Schmelzpunkt war $44^{\circ}$, sein Erstarrungspunkt $26^{\circ}$.

Das Fett der Periode II von demselben Aussehen wie das vorige hatte einen Schmelzpunkt von $38^{\circ}$, einen Erstarrungspunkt von $30^{\circ}$.

Diese beiden Portionen waren bei Verabreichung der gewöhnlichen Kost des K. u. K. Garnisonsspitales Nr. 1 in Wien erhalten worden.

Das Fett der Periode III ist bedeutend lichter gefärbt, flüssig, scheidet sich bei längerem Stehen in eine obere klare und eine untere von Krystallen durchsetzte Schicht und gibt in noch homogenem Zustande einen Schmelzpunkt von $34^{0}$ und einen Erstarrungspunkt von $26^{\circ}$.

Diese Portion wurde erhalten, als der Patient ausser

1) Ein Gemenge der isomeren Verbindungen ( $\alpha-, \beta-, \gamma$-Monoxystearinsäuren) ist deshalb anzunehmen, weil der Schmelzpunkt der vorliegenden Substanz niedriger war als der Schmelzpunkt einer dieser Säuren. 
gewöhnlicher Kost, zu deren Bereitung aber möglichst wenig Fett verwendet wurde, noch täglich eine Flasche (250 g) Lipanin erhielt. Ich habe schon oben angedeutet, dass der Vergleich der Eigenschaften des Fettes in den vorigen Perioden mit dem dieser Periode mit Entschiedenheit gegen die Annahme einer Secretion des Fettes spricht.

Das Fett der IV. Periode von demselben Aussehen. wie das der I. und II. stammt aus einer kurzen Versuchsperiode, in der der Patient $1 / 2 \mathrm{~kg}$ Walrat erhielt. In dieser Fettportion (ca. $80 \mathrm{~g}$ ) wurde der Nachweis des Cetylalkohols in folgender Weise versucht.

Nach dem Verseifen mit alkoholischer Kalilauge wurde die Seife in viel warmem Wasser gelöst und die Lösung mit Aether einige Male ausgeschüttelt. Der Aetherrückstand wurde in warmem Alkohol gelöst und durch Auskrystallisirenlassen nach wiederholtem Einengen von Cholesterin befreit. Es blieb so ein geringer Rückstand, der, nochmals verseift, aus alkalischer Lösung an Aether so gut wie nichts mehr abgab. Es war also Cetylalkohol trotz reichlicher Zufuhr von Walrat nicht nachzuweisen, ein Verhalten, das einerseits die Angaben von Munk und Rosenstein ${ }^{1}$ ) bestätigt, andererseits in Bezug auf unsern Patienten den Schluss gestattet, dass die die Fettassimilation betreffenden Qualitäten der Darmschleim-

- haut intact sein müssen.

$\mathrm{Zu}$ den im Folgenden beschriebenen qualitativen Bestimmungen wurde, wenn nicht anders bemerkt, das Fett der Periode I verwendet.

\section{Quantitative Bestimmungen.}

$\mathrm{Zu}$ diesen wurde das Fett im Wasserstoffstrom 3 Stunden bei $110-120^{\circ}$ getrocknet.

Die Elementaranalyse ergab :2)

C $76,59 \%$

H $11,88 \%$

$011,55 \%$.

1) 1. c.

2) $0,2878 \mathrm{~g}$ Fett gab $0,8157 \mathrm{~g} \mathrm{CO}_{2}$ und $0,3078 \mathrm{~g} \mathrm{H}_{2} \mathrm{O}$, 
Eine Probe des Fettes der Periode II gab1) :

$$
\begin{aligned}
& \text { C } 76,52 \% \\
& \text { H } 12,11 \% \\
& \text { O } 11,37 \% \text {. }
\end{aligned}
$$

Von den Constanten des Fettes wurden bestimmt2):

Specif. Gewicht bei $15^{\circ}$ C. : $0,920,0,905^{3}$ )

Schmelzpunkt .... $44^{\circ}$

Erstarrungspunkt. . . 26 $6^{0}$

Säurezahl . . . . . 3,508

Verseifungszahl ‥ 199,579

Aetherzahl. . . . . 196,071

Reichert-Meissl-Zahl . . 2,254

Hehnerzahl . . . . . 94,92

Jodzahl . . . . . 5 54,42

Schmelzpunkt der unlöslichen Fettsäuren $44^{\circ}$

Erstarrungspunkt > > 39,50

Verseifungszahl > $\quad$ > 201,785

1) $0,1492 \mathrm{~g} \mathrm{Fett} \mathrm{gab} 0,4193 \mathrm{~g} \mathrm{CO}_{8}$ und $0,1622 \mathrm{~g} \mathrm{H}_{8} \mathrm{O}$.

2) Diese Bestimmungen wurden nach dem ausgezeichneten Werke von Benedikt-Ulzer, Analyse der Fette und Wachsarten, Berlin 1897, vorgenommen.

Bestimmung der Säurezahl : 6,1025 g Fett verbrauchen $3,75 \mathrm{ccm}$. Lauge $(1 \mathrm{ccm} .=0,005714 \mathrm{~g} \mathrm{KHO})$.

Bestimmung der Verseifungszahl: 5,0859 $\mathrm{g}$ Fett werden mit $75 \mathrm{ccm}$. Lauge $(1 \mathrm{ccm} .=0,025909 \mathrm{~g} \mathrm{KHO})$ verseift; zur Retitrirung werden $27,7 \mathrm{ccm}$. Salzsäure $(1 \mathrm{ccm}$. entspricht $1,299 \mathrm{ccm}$. der Lauge) gebraucht.

Bestimmung der Reichert-Meissl-Zahl (nach Bondzynski und Rufi) und der Hehnerzahl: 5,0859 $\mathrm{g}$ Fett geben 4,8278 $\mathrm{g}$ unlösliche Fettsäuren, die zur Sättigung $37,6 \mathrm{ccm}$. Lauge $(1 \mathrm{ccm} .=0,025909 \mathrm{~g} \mathrm{KHO})$ brauchen; die löslichen Fettsäuren brauchen $2,25 \mathrm{ccm}$. Lauge $(1 . \mathrm{ccm} .=$ $1,019 \mathrm{ccm} \cdot \frac{\mathrm{n}}{10} \mathrm{KHO}=0,005714 \mathrm{~g} \mathrm{KHO}$ ).

Bestimmung. der Jodzahlen nach $\mathrm{Hübl}: \mathrm{Zu} 1,1893 \mathrm{~g}$ Fett werden $40 \mathrm{ccm}$. Hübl'sche Jodlösung $(1 \mathrm{ccm} .=0,02351 \mathrm{~g} \mathrm{Jod})$ zugefügt und zur Retitrirung 23,4 ccm. Hyposulfitlösung $(1 \mathrm{ccm}=0,533 \mathrm{ccm}$. Jodlösung) verbraucht. 1,3184 g unlösliche Fettsäuren, $40 \mathrm{ccm}$. Jodlösung, zur Retitrirung 19,7 ccm. Hyposulfitlösung. Cholesterin.

Bestimmung des Cholesterins: 6,7044 g Fett geben 0,1150 g

Bestimmung des Le cithins: 20,0 g Fett geben nach dem Verpuffen mit Soda und Salpeter $0,0154 \mathrm{~g} \mathrm{Mg}_{8} \mathrm{P}_{8} \mathrm{O}_{7}$, was einer Menge von $0,111958 \mathrm{~g}$ Lecithin entspricht.

3) Diese Zahl bezieht sich auf das flüssige Fett der Periode III. 


\section{$-445-$}

Acetylsäurezahl der unlöslichen Fettsäuren 201,5741)

Acetylzahl

12,1641)

Acetylverseifungszahl > > 213,7381)

Jodzahl

,

52,605

Aus diesen Bestimmungen berechnen sich folgende Werthe :

\begin{tabular}{|c|c|c|}
\hline ehalt des Fettes a & $\begin{array}{l}\text { an freien Fettsäuren : } \\
\text {, Neutralfett . . . } \\
\text {, Lecithin . . . . } \\
\text {, Gholesterin. . . } \\
\text {, Glycerin . . . . } \\
\text {, löslichen Fettsäuren } \\
\text {, unlösl. Fettsäuren . }\end{array}$ & $\begin{array}{c}1,680 \% 2) \\
95,987 \% \\
0,560 \% \\
1,715 \% \\
10,717 \% \\
95,573 \% \\
0,65 \% \\
94,92 \%\end{array}$ \\
\hline
\end{tabular}

Das mittlere Molekulargewicht der Ge-

sammtfettsäuren beträgt . . . . 268,64\%

der unlösl. Fettsäuren . $\quad 278,01 \%$

> löslichen Fettsäuren 133,8 \%

Die unlöslichen Fettsäuren bestehen aus

$58,4 \%$ Oelsäure

$31,3 \%$ Stearinsäure

$10,3 \%$ Palmitinsäure

oder unter Berücksichtigung der Oxyfettsäuren und der Myristinsäure näherungsweise :

$$
\begin{aligned}
58,4 \% & \text { Oelsäure } \\
6,6 \% & \text { Oxystearinsäure } \left.{ }^{8}\right) \\
25 \% & \text { Stearinsäure } \\
9 \% & \text { Palmitinsäure } \\
1 \% & \text { Myristinsäure. }{ }^{4} \text { ) }
\end{aligned}
$$

1) Bestimmung der Acetylsăure- und Acetylzahl der unlöslichen Fettsäuren (in dem Fett der Periode II bestimmt): 3,4001 $\mathrm{g}$ acetylirte Fettsäuren verbrauchen zur Neutralisation $23,2 \mathrm{ccm}$. Kalilauge $(1 \mathrm{ccm} .=$ $0,029542 \mathrm{~g} \cdot \mathrm{KHO}$ ). Zur Verseifung werden $50 \mathrm{ccm}$. derselben Kalilauge zugefügt. Retitrirung werden $43 \mathrm{ccm}$. Salzsäure $(1 \mathrm{ccm} .=1,13 \mathrm{ccm}$. Kalilauge) gebraucht.

2) Aus der Säure- und Verseifungszahl des Fettes berechnet; aus der Säurezahl allein unter der Annahme, das die freien Fettsäuren dasselbe mittlere Molekulargewicht haben wie die an Glycerin gebundenen, ergibt sich $1,738 \%$.

8) Aus Acetylzahl der Fettsäuren (Fett der Periode- II) berechnet.

4) Schätzungsweise. 


\section{Qualitative Untersuchung.}

$\mathrm{Zu}$ derselben wurden $200 \mathrm{~g}$ Fett mit alkoholischer Kalilauge auf dem Wasserbade verseift. Zweistündiges Kochen genügte zur vollständigen Verseifung. Der Seifenleim wurde mit viel Wasser verdünnt und zur Vermeidung der Bildung von Emulsionen die noch ca. $40^{\circ}$ warme Lösung einige Male mit Aether ausgeschüttelt. Diese Aetherauszüge hinterliessen nach dem Abdestilliren des Aethers einen tiefgelben, krystallinischen Rückstand unverseifbarer Substanz. Die wässerige Flüssigkeit wurde mit Schwefelsäure angesäuert und mit Aether ausgeschüttelt. Der nach dem Verdunsten des Aethers bleibende Rückstand wurde 6 Stunden lang im Dampfstrom destillirt und so in flüchtige und nicht flüchtige Fettsäuren getrennt.

Die saure, wässerige Flüssigkeit wurde mit Baryumcarbonat neutralisirt und eingedampft. Der Trockenrückstand wurde mit Alkohol extrahirt, die alkoholische Lösung eingegedampft, der Rückstand durch mehrmaliges Aufnehmen mit Alkohol, Filtriren und Eindampfen gereinigt und zuletzt im Vacuum destillirt.

Das Destillat ist eine schwach gelbgefärbte, dickliche, süsschmeckende Flüssigkeit. Sie löst Kupferoxydhydrat und Eisenhydroxyd, gibt mit saurem schwefelsauren Kalium, wie mit "Phosphorsäureandydrid erwärmt Acroleindämpfe, macht aus Borax Borsäure frei, reducirt nach längerem Kochen ammoniakalische Silberlösung und gibt die Reichl'sche Reaction (mit Pyrogallol und Schwefelsäure). Dadurch ist die Flüssigkeit als Glycerin identificirt.

Die unverseifbare Substanz, aus Alkohol mehrmals umkrystallisirt, lieferte blättchenförmige, durchsichtige Krystalle, die die Reactionen mit Salpetersäure, mit Chloroform und Schwefelsäure und die Liebermann'sche Cholestolreaction geben. Die aus Aether umkrystallisirte Substanz hat den Schmelzpunkt $147^{\circ}$. Die Elementaranalyse ${ }^{1}$ ) ergab :

1) 0,2116 g Substanz gab $0,6505 \mathrm{~g} \mathrm{CO}_{2}$ und $0,2451 \mathrm{~g} \mathrm{H}_{2} \mathrm{O}$. 


$\begin{array}{cc} & \text { für Cholesterin berechnet } \\ \text { C } 83,84 \% & 83,87 \% \\ \text { H } 11,90 \% & 11,86 \% \\ \text { O } 4,26 \% & 4,27 \%\end{array}$

Die vorliegende Substanz ist also Gholesterin.

Nach dem vollständigen Auskrystallisiren des Cholesterins blieb beim Verdampfen der Mutterlauge ein geringer unkrystallisirter Rückstand, der nochmals verseift wurde. Aus alkalischer Lösung liess sich dann mit Aether fast nichts mehr extrahiren. Cetylalkohol oder andere unverseifbare Substanzen ausser Cholesterin sind also nicht vorhanden.

Die flüchtigen Fettsäuren wurden nach dem Eindampfen mit $\mathrm{BaH}_{2} \mathrm{O}_{2}$ und Aufnehmen mit $\mathrm{H}_{2} \mathrm{SO}_{4}$-haltigem Wasser nochmals destillirt. Das Destillat zeigte einen deutlichen Schweissgeruch (Capron- oder Caprylsäure), mit Eisenchlorid eine kaum wahrnehmbare Rothfärbung (Essigsäure) und reducirte Silberlösung (Ameis ensäure). Wie oben angeführt, ist das Molekulargewicht der löslichen Fettsäuren 133,8. Das Molekulargewicht der Caprylsäure beträgt 144, das der Capronsäure 116, das der Ameisensäure 46. Nach diesen Zahlen wäre die Gegenwart der Caprylsäure wahrscheinlicher. Es war jedoch wegen der geringen Menge dieser Fettsäuren nicht möglich, sie genauer zu untersuchen, resp. durch die Barytgehalte ihrer Baryumsalze zu idendificiren. Aus diesem Grunde kann auch über die Gegenwart von Buttersäure, die man erwarten sollte, nichts ausgesagt werden.

Die nicht flüchtigen Fettsäuren wurden in warmem Alkohol gelöst und die nach dem Erkalten ausfallenden Antheile abfiltrirt und mit Alkohol gewaschen. Die Lösung wurde mit Bleizucker versetzt und, wie unten näher angeführt ist, weiterbehandelt. Der Rückstand aber wurde auf dem Filter mit warmem Alkohol gelöst und der beim Erkalten wieder ausfallende geringe Niederschlag. auf Arachinsäure geprüft, während die Lösung als ölsäurefrei sofort mit Magnesiumacetat fractionirt wurde. Um einer Esterification der eventuell vorhandenen Arachinsäure vorzubeugen, war nicht mit kochendem, sondern nur mit warmem Alkohol operirt worden. Der 
Schmelzpunkt des Ungelösten war übrigens $69,2^{0}$ und auch die erste Fraction desselben mit essigsaurer Magnesia gab denselben Schmelzpunkt, sodass Arachinsäure in nachweisbarer Menge nicht vorhanden war.

Die ersterhaltene alkoholische Lösung, die alle 0 elsäure enthalten musste, wurde zur Trennung derselben von den gesättigten Fettsäuren mit einer Lösung von essigsaurem Blei versetzt, mit viel Wasser verdünnt, der Niederschlag von fettsaurem Blei durch Erwärmen geballt, abfiltrirt, zwischen Filtrirpapier abgepresst und einige Tage im Vacuum über Schwefelsäure getrocknet. Dann wurde durch Behandeln mit kaltem Aether das ölsaure Blei von den Bleisalzen der gesättigten Fettsäuren getrennt.

Das ölsaure Blei wurde mit warmer $\mathrm{HCl}$ zerlegt, die Oelsäure öfter mit warmem Wasser gewaschen und in $\mathrm{NH}_{3}$ gelöst. Durch Ausfällen dieser ammoniakalischen Lösung mit Barythydrat wurde das Baryumsalz der Oelsäure dargestellt, das nach langem Auswaschen mit Wasser und Umkrystallisiren aus heissem Alkohol einen Baryumgehalt von 19,96\% $\%^{1}$ ) aufwies, der mit dem für 0 elsäure berechneten von $19,62 \%$ stimmt.

Die festen Fettsäuren wurden in alkoholischer Lösung mit essigsaurer Magnesia, und, wenn diese keine Fällung mehr gab, mit essigsaurem Blei fractionirt gefällt, die einzelnen Fractionen in der gleichen Weise weiter fractionirt, bis der Schmelzpunkt einer Fraction constant blieb. Auf diese Weise wurden folgende Fettsäuren isolirt :

1. Stearinsăure, Schmelzpunkt $69-69,2^{\circ}, 0,2105$ Substanz gab $0,5891 \mathrm{~g} \mathrm{CO}_{2}$ und $0,2407 \mathrm{~g} \mathrm{H}_{\mathrm{z}} \mathrm{O}$.
Gefunden :
C $75,94 \%$
H $12,71 \%$
Berechnet für $\mathrm{C}_{\mathbf{1 8}} \mathrm{H}_{38} \mathrm{O}_{\mathbf{8}}$ :
$0 \quad 11,35 \%$
$76,06 \%$
$12,68 \%$
$11,26 \%$
$0,2056 \mathrm{~g}$ des Silbersalzes gaben $0,0570 \mathrm{~g} \mathrm{Ag}$.
Gefunden 27,72\% Ag.
Berechnet 27,62\% ,

1) $0,3857 \mathrm{~g}$ ölsaurer Baryt gaben $0,1310 \mathrm{~g} \mathrm{BaSO}_{4}$. Der Erstarrungspunkt der erhaltenen Oelsäure war $6^{\circ}$, der Schmelzpunkt $16^{\circ}$. 
2. Palmitinsäure, Schmelzpunkt $62^{\circ}, 0,1767 \mathrm{~g}$ Substanz gab $0,4869 \mathrm{~g} \mathrm{CO}_{8}$ und $0,2010 \mathrm{~g} \mathrm{H}_{8} \mathrm{O}$.
Gefunden :
C $75,14 \%$
Berechnet für: $\mathrm{C}_{\mathbf{1 8}} \mathrm{H}_{\mathbf{3 8}} \mathrm{O}_{\mathbf{2}}$.
H $12,67 \%$
$75,00 \%$
$012,19 \%$
$12,50 \%$
$0,4156 \mathrm{~g}$ des Silbersalzes gaben $0,1226 \mathrm{~g} \mathrm{Ag}$.
$12,50 \%$
Gefunden 29,50\% Ag.
Berechnet $29,69 \%$,

3. Myristinsäure, Schmelzpunkt $54^{\circ}, 0,1376 \mathrm{~g}$ Substanz gab $0,3730 \mathrm{~g} \mathrm{CO}_{2}$ und $0,1540 \mathrm{~g} \mathrm{H}_{2} \mathrm{O}$.
Gefunden :
C $73,87 \%$
Berechnet für: $\mathrm{C}_{\mathbf{1 4}} \mathrm{N}_{\mathbf{8}} \mathrm{O}_{\mathbf{8}}$.
H $12,46 \%$
$73,68 \%$
$0 \quad 13,67 \%$
$12,30 \%$
$14,02 \%$
$0,1407 \mathrm{~g}$ Silbersalz gaben $0,0450 \mathrm{Ag}$.
Gefunden : $32,0 \% \mathrm{Ag}$.
Berechnet : $32,14 \%$,,

Stearinsäure wurde in bedeutend grösserer Menge als Palmitinsäure erhalten. Die Menge der Myristinsäure schätze ich auf etwa $1 \%$.

\section{Qualitative Reactionen.}

Diese wurden nach dem Verfahren von Crace-Calvert vorgenommen, wobei die unten beschriebenen Farbenveränderungen beobachtet wurden. $\mathrm{Zu}$ diesen Reactionen wurde das flüssige Fett der Periode III verwendet.

1. Natronlauge (spec. Gew. 1,340): dunkelgelb, fest.

2. Schwefelsäure ( , 1,475): schwachgelb.

3. Schwefelsäure ( , 1,530): röthlich.

4. Schwefelsäure ( $, 1,635)$ : dunkelrothbraun.

5. Salpetersäure ( \, 1,180): schwachgelb.

6. Salpetersäure ( , 1,220): schwachgelb-röthlich.

7. Salpetersäure ( , 1,330): röthlich.

8. Die nach 7 erhaltene Masse mit Natronlauge erwärmt: schmierige, hellbraune Masse.

9. Syrupöse Phosphorsäure: rothgelb.

10. Salpetersäure $(1,330)$ und Schwefelsäure $(1,345)$ aa part. aeq. : gelb.

11. Königswasser: gelb.

Hoppe-Seyler's Zeitschrift f. physiol. Chemie. XXX. 
12. Die nach 11 erhaltene Masse mit Natronlauge erwärmt: schmierige hellbraune Masse.

Reactionen nach Gläsner:

Rothe, rauchende Salpetersäure: eine rothe Zone, nach 1 Stunde unten ein grüner, darüber ein rosenrother Farbenring.

Concentrirte Schwefelsäure: an der Berührungsstelle braunroth.

Diese Reactionen nach Crace-Galvert nähern sich denjenigen, die die Thrane (rothe und braune Färbungen) geben, während nicht die leiseste Andeutung einer grünen Färbung, die den Pflanzenölen (auch dem zur Gewinnung dieser Fettportion in grösserer Menge eingeführten Olivenöl) zukommt, beobachtet wurde, ein Beweis, dass jene die Farbenreactionen bedingenden, charakterischen Verunreinigungen (Lipochrome etc.) des Nahrungsfettes die Darmwand nicht passiren, dass hingegen schon in der Darmwand die dem thierischen Fette eigenthümlichen Lipochrome etc. gebildet werden oder dem resorbirten Fett sich beimengen.

Anhangsweise will ich noch die auf die Seifen des Harnes, resp. Chylus sich beziehenden Zahlen hier anführen. Die aus einer Tagesmenge Harns gewonnenen Fettsäuren von $0,1403 \mathrm{~g}$ Gewicht verbrauchten $5,1 \mathrm{ccm}$. Kalilauge $(1 \mathrm{~cm}$. = 0,005808 g KHO) zur Neutralisation. Daraus berechnet sich ein mittleres Molekulargewicht von 266,2 für die aus den Seifen stammenden Fettsäuren, also ungefähr dasselbe, welches den Gesammtfettsäuren des Fettes $(268,64)$ zukommt.

Ebenso stimmt das mittlere Molekulargewicht der freien Fettsäuren mit dem der Fettsäuren aus den Glyceriden überein, wie sich daraus ergibt, dass der aus der Säure- und Verseifungszahl des Fettes berechnete Gehalt an freien Fettsäuren $\left(1,680^{\circ} \%\right)$ identisch ist mit demjenigen, der sich unter der Annahme, dass die freien Fettsäuren dasselbe mittlere Molekulargewicht, wie die aus den Glyceriden haben, aus der Säurezahl allein berechnet $(1,738 \%)$. Es sind also in den Seifen und den freien Fettsäuren des Chylus dieselben Fettsäuren in demselben Mengenverhältnisse, wie im Fett vertreten. 
Das menschliche Chylusfett besteht also aus den Glyceriden der 0el-, Stearin-, Palmitin- und Myristinsäure, von denen das Triolein den Hauptbestandtheil ausmacht. Das Tristearin beträgt das Dreifache des Tripalmitins, während -Trimyristin nur in kleiner Menge, die Glyceride flüchtiger Fettsäuren nur in Spuren vorkommen. Das Chylusfett enthält ausserdem eine geringe Menge (1,68\%) freier Fettsäuren, wie das auch Munk und Rosenstein angeben. Der Lecithingehalt desselben beträgt $0,56 \%$, der Cholesterin gehalt $1,715 \%$, also erheblich verschiedene Werthe, als die obengenannten Autoren fanden. Es mag das mit der Qualität der Nahrung zusammenhängen.

Es ist vielleicht interessant, die über die Zusammensetzung des Fettes des menschlichen panniculus adiposus bekannten Daten mit den entsprechenden des Chylusfettes zu vergleichen.

L. Langer ${ }^{1}$ ) fand, dass das Gemenge der Fettsäuren aus dem flüssigen Fette des Erwachsenen

$$
\begin{aligned}
& 89,80 \% \text { Oelsäure, } \\
& 8,16 \% \text { Palmitinsäure und } \\
& 2,04 \% \text { Stearinsäure }
\end{aligned}
$$

enthalte. Der Unterschied gegenüber dem Chylusfett besteht also darin, dass das Fett des panniculus oleinreicher und das Verhältniss des Palmitins zum Stearin verkehrt ist. Da das analysirte Chylusfett bei einer Nahrung gewonnen wurde, die mit den bei uns gebräuchlichen festen Fetten bereitet war, und der Patient dabei um $2 \mathrm{~kg}$ an Körpergewicht zunahm, die Nahrung also das zum Fettansatz nothwendige Material enthielt, so kann daraus direkt geschlossen werden, dass, wenn auch das Chylusfett in seiner Zusammensetzung vom Nahrungsfett abhängig ist (siehe oben), das als Reservematerial in denFettzellen aufgespeicherte Fett von der chemischen Zusammensetzung des vom Darm resorbirten Fettes unabhängig ist; ein neuer Beweis also dafür, dass das Fett nicht einfach

1) Ueber die chemische Zusammensetzung des Menschenfettes in verschiedenen Lebensaltern. Sitzb. der K. Akademie der Wissenschaften in Wien, Bd. 84, 1881. 
durch Infiltration, sondern durch einen secretiven Process in die Fettzelle gelange.

Wir haben endlich noch einen besonderen Befund zu erwähnen, nämlich das Monoxystearinsäurengemenge, das bei der Filtration des Harnfettes als im Fett ungelöst zurückblieb. Diese Verbindungen können künstlich aus der Oelsäure $\left(\mathrm{C}_{18} \mathrm{H}_{34} \mathrm{O}_{2}\right)$ erhalten werden (Geitel). Diese ungesättigte Säure liefert durch einfache Anlagerung von einem Molekül W'asser $\left(\mathrm{H}_{2} \mathrm{O}\right)$ die Monoxystearinsäuren $\left(\mathrm{C}_{18} \mathrm{~N}_{36} \mathrm{O}_{3}\right)$. Da in den als Nahrung verwendeten Fetten diese Oxysäuren nicht nachgewiesen wurden und jedenfalls, nach den Acetylzahlen dieser Fette zu schliessen, sicher nicht in solchen Mengen vorhanden sein können, so können wir nur annehmen, dass sie erst im Organismus offenbar aus der Oelsäure entstehen und zwar entweder im Darm durch Einwirkung von Fermenten oder Bakterien, oder aber in der Darmwand. Ich möchte das Erstere für das wahrscheinlichere halten, mich aut einen Befund Eberts $^{1}$ ) stützend, der im Adipocire Oxymargarinsäure nachwies.

Da nach Allem, was wir heute über die Adipocirebildung wissen, das Leichenwachs aus dem in der Leiche vorhandenen Fette unzweifelhaft durch die Wirkung von Bakterien ${ }^{2}$ ) entsteht - wenigstens fehlt für die Annahme einer anderen Entstehungsart jeglicher Anhaltspunkt -, so wird man wohl nicht fehlgehen, wenn man für die Oxysäure des Chylusfettes dieselbe Genese annimmt.

Zum Schlusse sei es mir noch gestattet, meinem hochverehrten Lehrer, Herrn Hofrath Prof. E. Ludwig, für manchen werthvollen Rath an dieser Stelle meinen besten Dank zu sagen.

1) Berichte der deutschen chemischen Gesellschaft, 1875.

2) Vergl. die jüngst erschienene Arbeit von Rubner. Ueber Spaltung und Zersetzung von Fetten und Fettsäuren im Boden und in Nährflüssigkeiten. Archiv für Hygiene, Bd. 38, Heft 1, 1900. 